\title{
RECENT IMPROVEMENTS IN THE U.S.N.O. COMBINED SOLUTION FOR EARTH ROTATION PARAMETERS
}

\author{
A. K. Babcock \\ U. S. Naval Observatory \\ Washington, D.C. 20390 \\ USA
}

\begin{abstract}
The USNO combined solution for Earth rotation parameters, commonly known as CORE has recently been made more sensitive to high-frequency signals in the various types of input data. This is particularly important in the estimation of the variation in UT1-UTC which has recently been shown to have real fluctuations over time intervals of less than a week. A second modification was introduced to tailor the data smoothing to the particular noise characteristics of the individual techniques. This has resulted in a redistribution of the weights of these techniques, and in improved time-resolution and accuracy of the CORE solution.
\end{abstract}

\section{THE CORE SOLUTION}

\subsection{What is it?}

CORE, the COmbined solution for the Rotation of the Earth, is an algorithm which produces estimates of the Earth orientation parameters (EOP) $x, y$, and UT1-UTC. The input for this algorithm is a variety of EOP estimates from different measurement techniques. The current set of input techniques includes $x, y$, and UT1-UTC from very long baseline interferometry (VLBI), produced by the U. S. Charting and Geodetic Service (C\&GS) as part of the International Radio Interferometric Survey (IRIS), x, y, and length of day (l.o.d.) from laser ranging to the Lageos satellite, provided by the Center for Space Research at the University of Texas, UTOY from the connected-element radio interferometer (CEI) operated by the National Radio Astronomy Observatory under contract with the Naval Observatory, $x$ and $y$ from Doppler ranging to Navy transit satellites, provided by the Defense Mapping Agency, and latitude and UT0 measurements obtained with photograpic zenith tubes (PZTs) operated by USNO.

The theory of the CORE solution has been discussed at previous meetings (McCarthy and Babcock, 1985) and that discussion will not be repeated now, but it may be helpful to review the main components of the process. As observations are read into the program, they are passed through a filter which is designed to detect errors in the data, to smooth the data if necessary, and to fill in short gaps in the data stream. Next, the data are adjusted for systematic differences between the individual technique and the CORE system (which is intended to be the reference frame defined by IRIS observations). At this point, the 
observations have been reduced to a series of estimates of variations, relative to a celestial reference system, of vectors originally defined in a terrestrial reference system, $\Delta B_{i}$, and associated weights, $w_{i}$. The program then compares these variations to adopted reference vectors and determines the changes in the Earth's orientation which are indicated by these observations. These changes in orientation are expressed in terms of estimates of $x, y$, and UT1-UTC. The program is run each week, as new data become available, and the values are made available in the NEOS (National Earth Orientation Service) Earth Orientation Bulletin and on computer-accessible files, including the G.E. Mark III system. The weekly results are generally based on optical, Doppler, Lageos, and CEI results and do not include any IRIS data. This is because the IRIS data generally do not become available until about a month after the epoch of observation. As soon as these data become available, CORE is run again for the period of the new data so that the resulting ERP estimates will be of the highest possible accuracy. These final results, which will be referred to from now on as "full-rate CORE", are not published but are available at any time by special request to the Earth Orientation Division of the USNO Time Service Department.

\subsection{Requirements for CORE}

The CORE solution was developed in order to meet the requirements for Earth rotation data of the U.S. Navy, as well as other services within the Department of Defense. Fortuitously, many of these requirements are shared by the astronomical, geodetic, and geophysical communities as well. These requirements include:

Quick-look estimates of ERP, provided at weekly intervals,

High-accuracy ERP for use in geophysical research,

Accuracy maintained at highest possible levels given current technology and knowledge,

Flexibility in the use of new techniques of observation and analysis,

Stability of the basic reference system, and

Reliability.

Some of these requirements conflict with each other. For example, flexibility and stability are not always compatible aims. We have found, however, that if accuracy is given the highest priority the other requirements are more easily met.

The routine availability of Lageos and VLBI data has introduced some new conditions into the problem of doing a combined solution. These techniques both are capable of detecting real variations in the Earth's orientation on timescales of a day or two (Robertson and Carter, 1985, Tapley et al., 1985). They both are capable of precisions in the submilliarcsecond range. Before these techniques were yielding ERP estimates routinely, it seemed reasonable to pass all the CORE input data through the same smoothing filter, and to disregard variations on timescales less than a week. Clearly, these practices are no longer acceptable.

\section{MODIFYING CORE}

\subsection{What changes were made?}

The last time we reported on the status of the CORE solution, at the Spring 1986 meeting of the American Geophysical Union, we announced several upcoming changes to the program. These included the use of IRIS daily determinations of UT1-UTC using single-baseline experiments, the use of exact epoch of observation whenever these were available for any technique, and the adjustment of the degree of smoothing for each data type, with the 
possibility of eliminating smoothing altogether for some techniques. All of these changes have since been made.

The IRIS single-baseline experiments are conducted by the stations at Westford, Massachusetts and Wettzell, in Bavaria. They yield estimates of the parameter, UT1-UTC, for almost every day on which a full, three-baseline IRIS experiment is not done. The data tapes from these experiments are sent to the Washington VLBI Correlator, located at and operated by USNO. The correlator output is then sent to C\&GS where the UT1-UTC estimates are derived. The entire process can be accomplished in about a week, under ideal conditions, and it is hoped that the resulting data will soon be available on a routine basis for the quick-look CORE solution, "quick-CORE". At present, these data are made available with the same delay as the full IRIS solutions, and are incorporated in the CORE solution as soon as possible, generally within a month of the experiments.

The exact epoch of "observation" is provided for the CEI results, the IRIS singlebaseline data, and the Lageos estimates. The 3-day means of Lageos observations are used, so the exact epoch is actually a mean epoch for the observations included in the mean. The CEI epoch is also a mean, but is based on measurements made over a single, 24hour period. The IRIS epoch is based on observations made over only about an hour and thus is the closest thing to an exact epoch of observation that is available. The full IRIS experiments are done every five days and include observations spread over several hours. C\&GS does not currently provide estimates of the precise epoch of the experiments, so the epoch assigned is 0 hours for the modified Julian date (MJD) corresponding to the civil date of the experiment.

Figure 1. Typical weight distributions in CORE. The full-rate solution is shown on the left and the quick-look solution on the right.
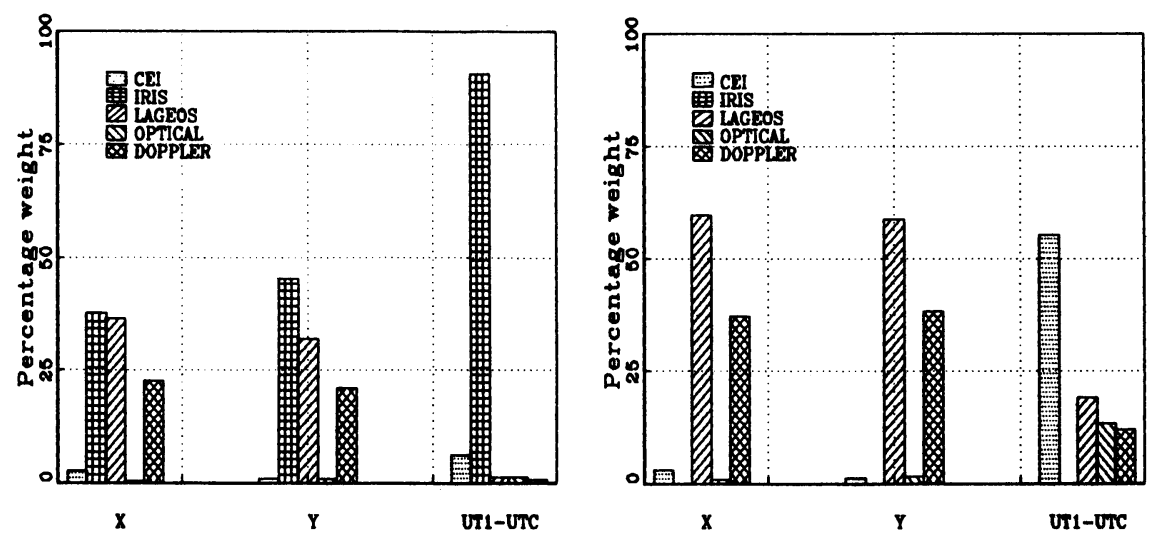

Finally, the smoothing constants were adjusted for each parameter provided by each technique. The aim of the adjustment was to minimize the rms of the residuals for each parameter relative to a full-rate CORE solution done for the same period of time. Clearly, this had to be an iterative process. Figure 1 illustrates two typical weight distributions, for full-rate CORE and quick-CORE. The full-rate CORE solution always includes IRIS data which receives about $90 \%$ of the weight for UT1-UTC and about $40 \%$ of the weight for $\mathrm{x}$ and y. Another $40 \%$ of the weight is taken by the polar motion estimates from Lageos ranging, 
and about $20 \%$ goes to Doppler. CEI data account for most of the remaining weight in the UT1-UTC solution. In the quick-CORE case, when IRIS data are not available, Lageos data assume most of the weight in $\mathrm{x}$ and $\mathrm{y}$ while CEI carries more than $50 \%$ of the weight in UT1-UTC. These weights should only be considered representative because very large redistributions in weight do occur from time to time, particularly when certain data are not available. One result of the adjustment of smoothing constants was that IRIS and Lageos data now receive no smoothing while the optical data are smoothed more than before. The smoothing of the Doppler and CEI data was not altered. As the smoothing constants were changed, the weight of IRIS and Lageos increased in the full-rate CORE solution.

\subsection{Results of Modifications}

The most important result was a significant improvement in accuracy, but we believe the time-resolution of the solution has also been increased. The improved time-resolution has been discussed at a previous meeting (McCarthy and Babcock, 1985) and I will not repeat that discussion here.

Table I shows the estimated accuracies of the CORE solution prior to the modifications described in this paper. At that time, the full-rate CORE accuracy was equivalent to the BIH Circular D values, but the accuracy of the UT1-UTC estimates in quick-CORE was considerably worse. Polar motion is a much smoother process and the errors do not vary significantly from one solution to another. These errors were estimated by comparing the different CORE solutions directly to the BIH values. Clearly, as further improvements were made to CORE, this method of estimating errors could no longer be used.

Table I. Estimated accuracies of CORE solutions.

\begin{tabular}{|l|c|c|}
\hline $\begin{array}{c}\text { C.O.R.E. } \\
\text { Solution }\end{array}$ & $\begin{array}{c}\text { Polar Motion } \\
\text { milliseconds of arc }\end{array}$ & $\begin{array}{c}\text { UT1-UTC } \\
\text { milliseconds }\end{array}$ \\
\hline Quick/before & \pm 3.0 & \pm 1.5 \\
Full / before & 3.0 & 0.5 \\
Quick/after & 2.5 & 0.5 \\
Full /after & 1.5 & 0.2 \\
\hline
\end{tabular}

The accuracy of the current full-rate CORE values is estimated by multiplying the internal error of the solution by a factor of three. The internal error is a measure of how well the techniques contributing to CORE agree with each other. Since VLBI data receive most of the weight in this solution, this is roughly equivalent to estimating the agreement between the rest of the contributors to CORE and VLBI. For the quick-CORE solution it was possible to calculate the rms of the estimates with respect to the full-rate CORE solution. The estimated accuracies of both solutions are shown in Table I. This analysis is based on CORE solutions covering the most recent three years.

\section{CONCLUSIONS}

This review of the CORE solution for Earth rotation parameters shows that it is a very reliable and highly-accurate time series which is useful for a wide range of scientific and technical applications, from satellite navigation to predicting Earth orientation. The current version of CORE is adequate for most present requirements, but it is clear that further improvement in accuracy and timeliness will be necessary. For this reason, continued effort 
will be put into incorporating more high-precision techniques, into refining the methods used to "clean" the input data (i.e. smoothing and interpolation methods), and alternate schemes for the combination of different types of geodetic measurements will be investigated. Our intention is always to provide the most accurate possible estimates of the the Earth's orientation with the shortest possible delay.

\section{REFERENCES}

McCarthy, Dennis D. and Babcock, Alice K., 1985, 'The U. S. Naval Observatory C.O.R.E. Solution During Project MERIT', in Proceedings of the International Conference on Earth Rotation and the Terrestrial Reference Frame, July 31 - August 2, 1985, Columbus, Ohio.

Robertson, D. S. and Carter, W. E., 1985, 'Earth Orientation Determinations from VLBI Observations', op. cit..

Tapley, B. D., Eanes, R. J., and Schutz, B. E., 1985, 'Analysis of Earth Rotation from Lageos SLR Data', op. cit..

\section{DISCUSSION}

Pâquet: For Polar Motion your solution attributes to the different techniques weights which are very different that those used in the BIH solution. For example, Doppler has a weight of 25 in your solution against few percents in the BIH solution. Could you explain the origin of this difference?

Reply by Babcock: The weights of the contributing techniques vary considerably depending on the particular combination of techniques used in a solution. Doppler receives a lot of weight in our quick-look solution which does not include VLBI data, but relatively little when VLBI is included.

Kaplan: How often are your systematic corrections re-evaluated?

Reply by Babcock: At least once a year, and more often if a new contributor is added to the solution or if we learn of some change in the characteristics of one of our sources of data.

Eanes: Why do your figures for the accuracy of the CORE solution show 4 mas for $x$ and $y$ when other intercomparisons conclude that the SLR data you use have 2 mas accuracy?

Reply by McCarthy: That viewgraph showing the accuracy of $x, y$, and UT1-UTC reflects the fact that Lageos 5-day solutions were used in the solution. The accuracy is improved with 3-day Lageos values.

Dickey: You compare your "quick-CORE" and "full-rate CORE" solutions with the BIH solutions. What is the time lag or data delay for each of these solutions? When one compares the accuracies of solutions, one must consider those with similar time delays.

Reply by Babcock: The quick-CORE solution is produced each week and usually is complete to within 3 to 6 days of time of publication, roughly comparable to BIH rapid service. It takes about a month to obtain all the data for the full-rate solution, which is produced with a delay comparable to BIH Circular D values. 